\title{
Semiotic Interpretation of Lotka-Volterra Model and its Usage in Knowledge Management
}

\author{
Kirill E. Evdokimov ${ }^{1}$, Valery G. Kuznetsov ${ }^{2}$, Irina V. Melik-Gaykazyan ${ }^{3, a}$, Maria V. Melik-Gaykazyan ${ }^{1}$, \\ ${ }^{1}$ National Research Tomsk Polytechnic University, 30 Lenin Avenue, Tomsk, 634050, Russia \\ ${ }^{2}$ Lomonosov Moscow State University, 27/4 Lomonosov Prospect, Moscow, 119991, Russia \\ ${ }^{3}$ Tomsk State Pedagogical University, 60 Kievskaya st., Tomsk, 634061, Russia
}

\begin{abstract}
Convergence of NBICS-technologies makes relevant the exact definition of objective goals' spectrum, which pursued this self-organizing system of technologies. Authors consider the objective goals of this system of technologies as "semiotic attractors" and the tasks related to knowledge management at the NBICS-technologies niche as management of competition between the goals, which cause processes of creation, transmission, reception, usage and duplication of the new knowledge. Competitive interaction of these goals (and their symbolizations) were researched on the grounds of Lotka-Volterra model. The original interpretation of Lotka-Volterra model is posed on the basis of stated interconnection between the stages of complex systems' non-linear dynamics, this self-organization's information mechanisms and the semiotic results of information processes' stages. This synthesis of synergetic, cybernetic and semiotic paradigms is implemented on the grounds of A. N. Whitehead process philosophy. Semiotic interpretation of the model allowed determining the order of goals' conversion and defining the stages of dynamics at which this transformation by means of knowledge management is constructive.
\end{abstract}

\section{Introduction}

Knowledge management initiated emergence of the new form of futurology. This statement confirms convergent nature of NBICS-technologies' dynamics [1-2]. Convergence supposes converging of evolution trajectories to some single "point" in the future. This expected "singularity" is a result of management's special efforts [3-4], that stimulates conducting researches based on the inter-disciplinary paradigm, embodiment of obtained intellectual actives in the new technologies and searching means of its implementation in all areas of modern life. "Life" (not "reality", for instance) is used knowingly. It expresses specifics of NBICS-technologies. Behavior of the NBICStechnologies system is similar to biological systems' dynamics and so sometimes, it is described using modifications of Lotka-Volterra model, in particular for predicting competitive advantages of different innovative strategies that should be implemented within a single organization or association of organizations [57]. But initially this model was created for researching population dynamics within the limits of the "predator- prey" system [8-9]. Lotka-Volterra model is adequate for solving 2 related tasks: a) definition of such state of competitive interaction that gives sustainable advantage to one of parties, b) predicting the moment in future when such sustainable advantage will come irreversibly. These circumstances provide relevancy of the model for producing managerial strategies related to convergent technologies which explain development of its modifications up to the present time [for instance: 1012]. A new store of managerial methods and increasing power of NBICS-technologies determine achievement of "singularity" in the future. The problem is eccentricity of image of such near future [13-14]. It make actual to research mechanisms of symbolization of managerial goals to clarify is it possible to adjust the future states that will be achieved inevitably. In fact, such setting of research objectives is related to necessity of developing special semiotic means of management [15], that currently remains only its "ornamental add-on" [16]. We propose the semiotic interpretation of Lotka-Volterra model as a possible tool for predicting results of competition between goals' symbols and optimizing management of symbolization of goals' processes.

\footnotetext{
$\overline{{ }^{a} \text { Corresponding author: melik-irina } @ y a n d e x . r u ~}$
} 


\section{The conceptual grounds of semiotic interpretation of Lotka-Volterra model}

Knowledge management is management of processes which reason consists of its goal. Creation of knowledge, exchange of it, its storage and usage are always prompted by a specific goal. Moreover, this goal is individual but the whole path from creation to application of knowledge is a collective process. That is why the teleological process is the matter of knowledge management while its primary task is to place the goal that cause co-operative effects of self-organization. Management's task is always to develop and implement the optimal way to reach the goal. We ask: Can the very way of placing goal optimize management itself? In fact, it is a question about creation of "semiotic attractor" for the controllable system [17]. Such statement of a question requires refusing from the cybernetic paradigm, which treat knowledge management as management of the "information processing system" realized by a human or an institution [16]. It would be better to escape absolute refusal from the cybernetic paradigm because knowledge exchange processes are similar to information transmission processes. Besides that, within this paradigm they found analogues in dynamics of biological and artificial systems which provided elaborating the most methods of complex systems' management. The limits of the cybernetic paradigm's applicability become clear while modeling spontaneous process of information generation or any occasions similar to scientific discoveries. These occasions do not obey to the deterministic laws but do not contradict them. Such understanding of information generation was offered within the synergetic paradigm [18]. Thus they founded interpretation of information phenomenon as an incidental and memorized choice of a single alternative from a multitude of possible alternatives. It became the foundation of the valuable information evolution conception (i.e. the one that increases probability of reaching the goal) as self-organization of complex open systems. This interpretation guaranteed application of Lotka-Volterra model for description of competition between goals in self-organizing systems of any nature [18]. But in this seemingly realized synthesis of synergetics and information theory one thing remains unclear, namely selecting a goal does not mean selecting a way of reaching a goal and selecting a way of reaching a goal does not mean reaching a goal is possible. In other words, information turned into the result of the process of its generation, and information, turned into results of creating an operator for its usage, are not the same "thing", are not the same "data" or "material", though they often identify information this way. Resulting differences of all stages of information process (generation, encoding and transmission, storage and reception, building operator and re-duplication of its work's results) constitute semiotic forms in which they are embodied. It is worth saying it was Ch. S. Peirce who chose classes of results of represented signs of fundamentally different processes. All above-mentioned factors give the basis for the fundamental opportunity to synthesize the cybernetic, synergetic and semiotic paradigm for modelling processes managing symbolizations of goals. It should be observed that our concept of such synthesis was initialized by Whithehead's philosophy of the process [19], in which teleological processes correspond to the modern conception of the attractors' role; conceptual theses on conditions of events' occurrence meet synergetics' deduction on the role of bifurcations; and his thesis about management as an ability to tell time for organizing "revolution in symbolism" [20] leaves the theory of management behind.

Figure 1 represents the proposed synthesis schematically. Please note initially this scheme was elaborated for research of self-organizing scientific systems [21], and was used for semiotic diagnostics of social goals' transformation much later (for example in [22-23]).
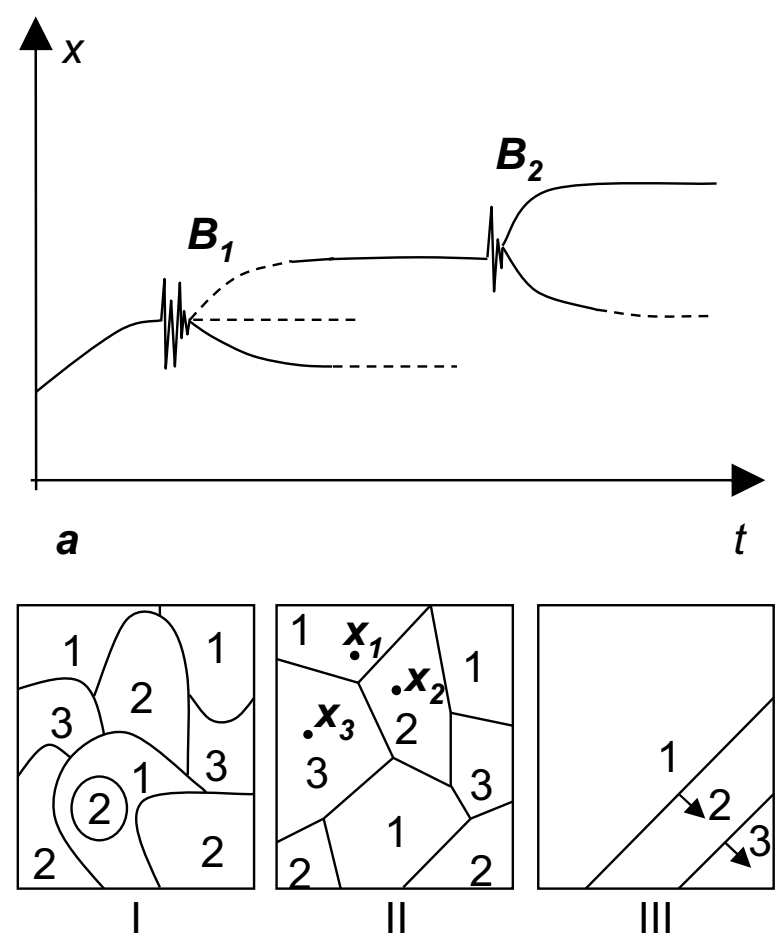

b

Fig. 1 Correspondences between phases of self-ogranization and stages of information processes: a) bifurcation diagram; b) phases of valuable information's evolution - «mosaic» (I), «parquet» (II), transition to "the pure" cluster (III), based on solution of Lotka-Volterra model [18]; Parts of quickly changing $x$ at $B_{1}$ and $B_{2}$ area denote pre-bifurcation states. Dotted line at the "branches" of the bifurcation diagram show that a definite decision may gain (or lose) actuality

Let us highlight correspondences between primary phases of self-organization and stages of information and semiotic dynamics:

1. the system overcomes the chaotic state and generates information as selection of a "quitting chaos" variant and forming the spectrum of goal's semantic expressions, which corresponds to situation of new knowledge creation which value may be determined only retrospectively; 
2. fixation of selected new order's variants, information encoding as a condition of its storage and/or transmission, which corresponds to presentations of scientific achievements;

3. shaping of the new structure levels of the system (complication in case of self-organization or simplification in case of self-disorganization), information transmission and creation of images for fixation of new structureness, which corresponds to the process of knowledge exchange;

4. "memory" about sustainable states (a dotted line in Picture 1), information storage (the process continues until its media exist) and shaping of thesaurus, which corresponds to preserving of knowledge and the educational content;

5. shaping of attractor structures (approximation of $\mathbf{B}_{2}$ in Picture 1,a), creation of an operator as a method of reaching a goal (stage II in Picture 1,b) and shaping of styles, which corresponds to usage of knowledge;

6. achieving an attractive state (a continuous line after $\mathbf{B}_{2}$ in Picture 1,a), re-duplication of information (stage III in Picture 1,b), "revolution in symbolism", which corresponds to support of knowledge.

It is clear all listed stages and phases do not replace each other, have different duration and their trajectory is variable. Even pictured in a schematic way, interrelation of self-organization's information mechanisms and phases of semiotic dynamics demonstrate the key role of goals' competition that are peculiar to structural elements of the complex systems. It helps to optimize management on the basis of determining the place and the time when goals' symbolization was corrected. On these grounds there were planned numerical experiments with Lotka-Volterra model, in which competing elements were the pursued goals.

\section{Results of calculation and analysis}

In this section we introduce a model [24] describing dynamics of number density of elements $n_{i}$. Here subscript $i$ corresponds to a certain element carrying $i$-th type of information, and number densities are functions of phase coordinates and time. The model is based on generalized Lotka-Volterra model $[17,18]$, and the following processes are considered: 1) competition of $j$ th element and $i$-th element with rate $\left(-b_{i j} n_{i} n_{j}\right)$, where $b_{i j}$ is a competition coefficient; 2) self-competition with rate $\left(-a_{i} n_{i}^{2}\right)$, where $a_{i}$ is a corresponding coefficient; 3) autocatalytic reproduction with rate $n_{i} / \tau_{i}$, where $\tau_{i}$ is characteristic time of self-reproduction; 4) an element number distribution in the phase space due to diffusion with rate $\nabla\left(D_{i} \nabla n_{i}\right)$, where $D_{i}$ is a diffusion coefficient for $i$-th element. Phase space coordinates and time are dimensionless.

The model is realized in the following system of equations

$$
\begin{gathered}
\frac{\partial n_{i}}{\partial t}=\nabla\left(D_{i} \nabla n_{i}\right)+\frac{n_{i}}{\tau_{i}}-a_{i} n_{i}^{2}-\sum_{i \neq j} b_{i j} n_{i} n_{j}, \quad i, j=\overline{1, k} \\
n_{i}(t=0, \mathbf{r})=\sum_{j=1}^{m} \alpha_{j} e^{-\beta_{j}\left(\mathbf{r}-\mathbf{r}_{j}\right)^{2}}, \quad i=\overline{1, k} \\
\left.n_{i}\right|_{\partial V}=0, \quad i=\overline{1, k}
\end{gathered}
$$

where $k$ is a maximum number of elements. Here equation (2) describes initial distribution of elements. In this work we choose random distribution of $m$ Gaussshaped peaks in the phase space, where $\alpha_{j}, \beta_{j}$ are peak parameters, and $\mathbf{r}_{j}$ is a random vector in the phase space. Equation (3) describes boundary conditions of zero number density, where $V$ is a phase space volume under investigation, and $\partial V$ is the boundary of this volume. Element number density dynamics is visualized by following algorithm: each element has its corresponding color and if $i$-th element dominates in some position of the phase space, the $i$-th color is assigned to that position.

In this work we consider the three-element model with two spatial variables and one time variable. The system (1-3) was solved numerically using Wolfram Mathematica. Figure 2 represents the results of calculations with the following parameter values: $D_{1}=D_{2}=D_{3}=0.05 ; \quad \tau_{1}=1.1 ; \quad \tau_{2}=1.15 ; \quad \tau_{3}=1$; $a_{1}=a_{2}=a_{3}=0.1 ; b_{12}=b_{13}=b_{21}=b_{23}=b_{31}=b_{32}=5$. a) $\mathrm{t}=0$

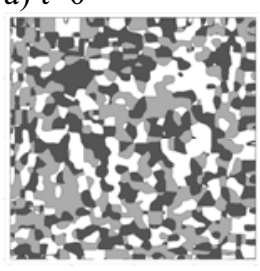

d) $\mathrm{t}=0.1$

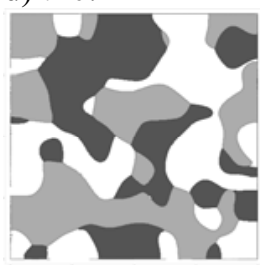

g) $\mathrm{t}=1$

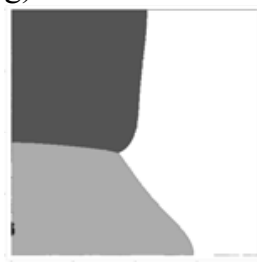

b) $\mathrm{t}=0.01$

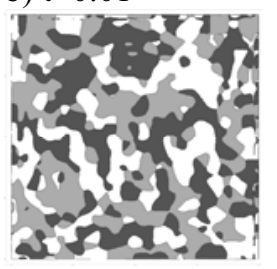

e) $\mathrm{t}=0.2$

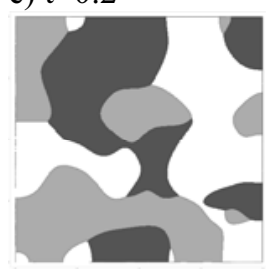

h) $\mathrm{t}=2$

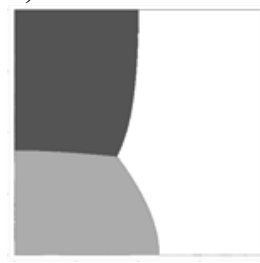

c) $\mathrm{t}=0.05$

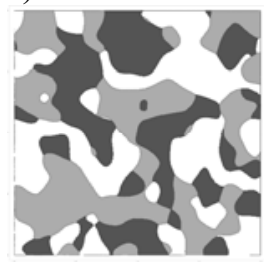

f) $\mathrm{t}=0.5$

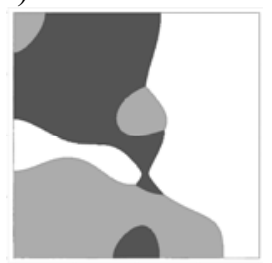

i) $\mathrm{t}=10$
Fig. 2 Distribution of element domination over the phase space and time. Charts a)-i) correspond to different moments of time. Abscissa and ordinate correspond to spatial variables. Dark gray color corresponds to element 1 , light gray to element 2 and white - to element 3.

The analysis of results shows that the considered system passes through the following stages: the 
"mosaic" stage, when all phase space is covered by a "mosaic" of small pure clusters of certain elements (Figure 2b); the "parquet" stage, when the cluster boundary curvature and the number of clusters decreases (Figure 2h); the pure cluster stage, when one element dominates over all phase space (Figure 2i). Parameter values, initial and boundary conditions may influence dynamics only quantitatively, and general sequence does not change.

It should be pointed out that at the "mosaic" stage it is impossible to predict what element will dominate if system parameters are unknown. We illustrate this thesis by results of calculations with the same initial condition as presented in Figure 2 and with different parameter values (Figures 3, 4).

a) $\mathrm{t}=0.1$

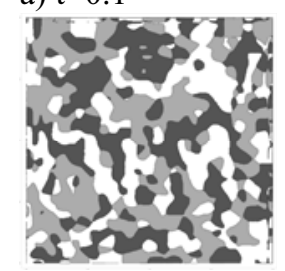

b) $t=2$

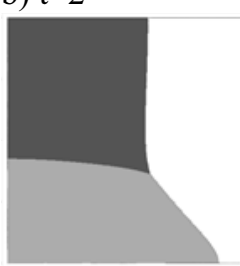

c) $t=10$

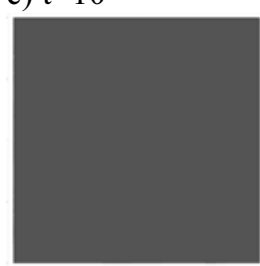

Fig. 3 Time and space dynamics of element distribution leading to domination of element 1 (dark gray). Parameter values:

$D_{1}=D_{2}=D_{3}=0.05 ; \quad \tau_{1}=1 ; \quad \tau_{2}=1.05 ; \quad \tau_{3}=1.15 ;$

$a_{1}=a_{2}=a_{3}=0.1 ; \quad b_{12}=b_{13}=b_{21}=b_{23}=b_{31}=b_{32}=5$

Stages: a) "mosaic", b) "parquet", c) pure cluster

a) $\mathrm{t}=0.1$

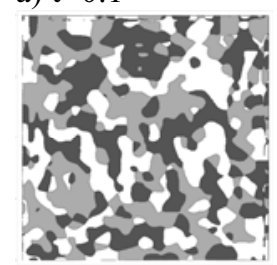

b) $t=2$

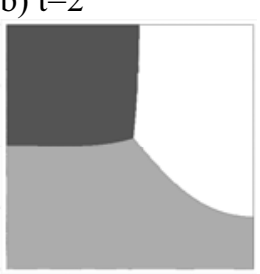

Fig. 4 Time and space dynamics of element distribution leading to domination of element 2 (light gray). Parameter values:

$D_{1}=D_{2}=D_{3}=0.05 ; \quad \tau_{1}=1.2 ; \quad \tau_{2}=1 ; \quad \tau_{3}=1.25 ;$ $a_{1}=a_{2}=a_{3}=0.1 ; b_{12}=b_{13}=b_{21}=b_{23}=b_{31}=b_{32}=5$

Stages: a) "mosaic", b) "parquet", c) pure cluster

It should be noted that at the final stage three different elements dominate (Figure 2i, Figure 3c, Figure 4c) despite the fact that at the "mosaic" stage the distribution of elements is virtually the same (Figure $2 b$, Figure 3b, Figure 4b).

\section{Conclusion}

The results of numerical experiment visualized three sustainable phases reached by the system inevitably having arbitrary defined conditions of an initial chaotic stage: "mosaic", "parquet" and "the pure cluster". We assess these sustainable phases as structures similar to attractors at the definite stages of non-linear dynamics. Among media (i.e. followers of some goal) competition the winner can be predicted for such transitions of configurations as "mosaic" - "parquet" and "parquet" "the pure cluster", but cannot be predicted fundamentally for the "mosaic" - "the pure cluster" transition. The role of autocatalytic reproduction and the diffusion dynamics' rate during a "mosaic" period shows that domination of the media type is provided by the rate of short-term goals expansion. Observing the same indices during transition from the "mosaic" to "parquet" stage revealed these goals are not essential. The same was true for the "parquet" to "the pure cluster" transition. Hence, each transition corresponds to a definite format of goals' symbolization and its replacement of the format that should be managed. In semiotic approximation the obtained results can be presented in the following way:

1. at the "mosaic" stage the one whose goal fixes semantics or unambiguously answers the question "what to do?" has competitive advantages;

2. during transition to the "parquet" stage the one whose goal has accented syntactics or contains the answer to the question "which way to do it?" will win;

3. the transition to "the pure cluster" stage is devoted to reaching the goal that reveals pragmatics of action or answers such questions as "why to do it?", "what for to do it?", "what was the real reason of this action?"

Experimenting with the time frame while modeling on PC allows construing the periods between stages of stages ("mosaic", "parquet", "the pure cluster") as an irreversible sequence "today", "the day after tomorrow", "the distant future". The fundamental randomness of transition from the "mosaic" phase to "the pure cluster" phase, semiotic approximation of PC-based experiments and the revealed rate of transitions allow making the conclusion: it is the symbolism that clearly expresses the order of actions for "the day after tomorrow" and never verbalizes goals of "the distant future" and that can turn into a "semiotic attractor". Asymptotic goals produced "today" possess only the power of utopia.

Semiotic interpretation of Lotka-Volterra model allowed determining the order of goals' conversion and defining at which stages of dynamics this transformation is constructive. The calculations made and visualization obtained may be used to diagnose the effectiveness of the semiotic expression of knowledge management goals.

\section{Acknowledgment}

The results were obtained in the framework of RFBR project №14-06-00440 “Development of Ways to Use Research Methods of Nonlinear Dynamics for the Detection of Social System of Self-organization Attractors", RHF project № 15-03-00598 "Bioethics: Creation of the New Symbolism” and project № 155 "Methodology of the Modeling the Semiotic Mechanisms of Management of Educational Systems Nonlinear Dynamics" of the State assignment for "Tomsk State Pedagogical University". 


\section{References}

1. M. Roco, W. Bainbridge, Converging Technologies for Improving Human Performance: Nanotechnology, Biotechnology, Information Technology and Cognitive Science (Dordrecht: Kluwer Academic Publishers, 2003)

2. M.V. Kovalchuk, Nanotechnologies in Russia, 6, 1 (2011).

3. NRC, Research Universities and the Future of America: Ten Breakthrough Actions Vital to Our Nation's Prosperity and Security (Washington: The National Academies press, 2012).

4. NRC, Convergence: Facilitating transdisciplinary integration of life sciences, physical sciences, engineering, and beyond (Washington: The National Academies press, 2014).

5. A.S. Chakrabarti, Physica A, 442, 214 (2015)

6. A. Marasco, A. Picucci, A. Romano Technol. Forecast. Soc., 105, 49 (2016)

7. H.-T. Wang, T.-C. Wang Technol. Forecast. Soc., 106, 37-44 (2016)

8. A.J. Lotka, Elements of Physical Biology (Baltimore: Williams and Wilkins, 1925)

9. V. Volterra, Animal Ecology (New York: McGrawHill, 1931)

10. J. Knebel, T. Krüger, M. F. Weber, E. Frey, Phys., Rev. Lett. 110, 168106 (2013)

11. G. W. A. Constable, A. J. McKane, Phys. Rev. Lett. 114, 038101 (2015)

12. A. Zilburg, P. Rosenau, J. Phys. A: Math. Theor, 49, 095101 (2016)

13. R. Kurzweil, The Singularity Is Near (N. Y.: Viking, 2005).

14. P.D. Tishchenko, Probl. Phil, 8, 181 (2014)

15. L. Sjarbaini, R.J. Jorna, Semiotica, 195, 169 (2013)

16. G. Rossolatos Int. J. Market. Semiot, 2, 23 (2014)

17. I. Melik-Gaykazyan, K. Evdokimov, M. MelikGaykazyan, International Society for Engineers and Researchers: 2-nd International Conference. Singapore: Institute of Research and Journals, 52 (2015)

18. D.S. Chernavski, Synergetics and Information (Moscow: Znanie, 1990)

19. A.N. Whithehead, Process and Reality (New York: The Macmillan Company, 1929)

20. A.N. Whithehead, Symbolism, Its meaning and Effect (New York: The Macmillan Company, 1927)

21. I.V. Melik-Gaykazyan, M.V. Melik-Gaykazyan, V.F. Tarasenko, The methodology of modeling the nonlinear dynamics of complex systems (Moscow: Phys. and Math. Literature Publishing Company Russian Academy of Sciences, 2001)

22. T.V. Mescheryakova, I.V. Melik-Gaykazyan, Scholae. Ancient Phil. Class. Trad, 9, 36 (2015)

23. M. Gorbuleva, I. Melik-Gaykazyan, M. MelikGaykazyan, Sociol. Stud, 4, 152 (2016)

24. K. Evdokimov, E. Melik-Gaykazyan, TSPU Bulletin 11, 193 (2013) 